\title{
THE CONSTITUTION OF SPECIAL RELATIVITY
}

\author{
Tapan Kumar Ghosh \\ River Research Institute, West Bengal, Nadia, 741246, India \\ gtkrri@gmail.com
}

\begin{abstract}
Lorentz transformation relations of special relativity are generally derived for light like intervals in almost all texts. The procedure assumes invariance of spherical wave front equation in all inertial frames. This paper deals with the derivation of the transformation relations of event coordinates together with Doppler formula from a single treatment by constructing identical length and time standard under identical conditions in compared frames. To meet this end critical review of the postulates of Special Relativity in the perspective of equivalence of length, time and mass standard followed by the meaning of phase of clock in any inertial frame is presented. It has been shown that special relativity is in reality a supreme creation of human mind where identical length and time standards being independent of the perspective are not naturally given but had been constructed under identical experimental condition in a scientific way so that observations from different frames can be compared using same system of units.
\end{abstract}

\section{Keywords}

Equivalence of frames, equivalence of base units, Special Relativity, Postulates.

\section{Academic Discipline And Sub-Disciplines}

Physics theoretical.

\section{SUBJECT CLASSIFICATION}

Relativity.

\section{APPROACH}

Historical and heuristic approach in logical foundation of Special Realativity.

\section{INTRODUCTION}

Special theory of relativity is already one hundred and ten years old but the marvels of the architecture designed by Einstein still remains an example of the creativity of supreme mind. The whole edifice was built upon a totality of eight postulates among which the postulates of $c$ invariance and the invariance of laws of physics are the starting ones. But it is astonishing enough that the postulate of $c$ invariance actually contains two postulates. It was a master trick of Einstein. The first among these two is that the electromagnetic pulse speed is ultimate speed limit in any inertial frame; the second is that same numerical value with same unit namely " $\mathrm{ms}^{-1}$ " can be used in all frames provided we can construct equivalent or identical clocks under identical circumstances in compared frames. As regards the invariance of laws of physics it is clear from Special Relativity that Einstein considered Maxwell's laws as invariant laws. But the question is that if Maxwell's laws of electrodynamics are regarded as 'laws' then at first sight it is not clear what does invariance of Maxwell's laws mean in addition to c invariance. The reason is that Maxwell's laws in free space produce electromagnetic pulse propagation with speed $c$ and that has already been taken as invariant. It appears as if the invariance of Maxwell's laws is an exaggeration. But each and every brick of the construction was precisely chosen by Einstein. Einstein was master architect. He cautiously had chosen the caption of his paper of 1905[4]. It was "On the Electrodynamics of Moving Bodies". To him laws electrodynamics meant not only four laws of Maxwell but also Lorentz force relation $\mathbf{F} / \mathbf{q}=\mathbf{E}+\mathbf{v x B}$. So postulate of invariance of laws of physics again essentially contains three postulates - one is 'form invariance' or covariance of Maxwell's laws; the second is covariance of Lorentz force relation and third is charge invariance. From these three, one can determine how force transforms from one frame to another. Once this is known, it can be used in mechanics and laws of mechanics can be reformulated to take a covariant form. This was Einstein's programme. This paper deals with the delicacy in the matter of postulates chosen by him. Other postulates namely the equality of length standard perpendicular to the relative motion between two frames, simultaneity of all clocks on that perpendicular axis and the homogeneity of space and time required for conservation of linear momentum and energy follow in his theory as necessary ingredients so that 'event' can be compared from different inertial frames with equivalent length, time and mass standard under identical experiments.

\section{MEANING OF INVARIANCE OF c FROM EQUIVANENCE OF LENGTH AND TIME STNDARDS}

Einstein's special relativity is based upon two postulates -invariance of c in all inertial frames and invariance of Laws of Physics in all inertial frames. The question is why did Einstein put two postulates in his theory? Is invariance of light speed (c) in free space is not the law of nature? If speed of light in free space is c as observed in one inertial frame then it ought to be same in all inertial frames as demanded by invariance of Maxwell's laws of electrodynamics. Is invariance of $c$ a separate postulate from the invariance of Maxwell's equations? The situation is almost identical with Newton's 
formulations of the three laws of mechanics. His first law i.e, law of inertia seems a mere conclusion from his second law in the context $F=0$. But Newton gave the law of inertia the status of first law. He was man of few words. He formulated law of inertia to find such frames where 'inertia' as a property of material particle defined by first law holds. Such frame is called inertial frame. If such a frame exists then only his force law or second law will hold in that frame. So law of inertia is actually a definition of inertial frame. Almost same situation is there as regards the two starting postulates of special relativity. It is an established fact that value of $\mathrm{c}$ is independent of the relative motion of source with respect to detectors. It means one can never take over $c$ by any kind of speed of the inertial frame. So speed of light in free space must be an ultimate speed limit. So a light pulse if emitted from the head light of a stationary train is $c$ as measured by ground observers then it is also $c$ to the ground observers if pulse were again emitted from the head light of a moving train. If this were not true then we on the earth bound frame could observe different values of $c$ emitted from different planets or stars of different motional states. But does it give any guarantee that value of $\mathrm{c}\left(2.99792458 \times 10^{8} \mathrm{~ms}^{-1}\right)$ will also remain same to the observers in another frame i.e., in a moving train? It is true that if light pulse is in flight as observed in one frame it keeps on going in all inertial frames. But the answer is clearly not in affirmative if we do not use identical length and time standards in both frames. So invariance of $c$ in all inertial frames is a primary requirement for construction of identical length and time standards. Without this identity of length and time standard (in SI they are metre and second) speed of light $\mathrm{c}$ in other inertial frame is to be written as $\mathrm{c}^{\prime} \mathrm{m}^{\prime} \mathrm{s}^{\prime-1}$. In an equivalent way we can say if identity of length standards in all frames are ensured and if $c$ is taken invariant in all frames then we can guarantee for identity of clocks or identity of time standards in both frames. In this context it is worth mentioning that the epoch making experiment on Michelson and Morley does not directly prove invariance of $c$ in all frames rather it disproves the existence of absolute frame or ether frame where light was supposed to move with speed c. Michelson-Morley experiment clearly proved that speed $c$ measured in our earth bound laboratory frame does not change at all with the motional state of the laboratory frame with respect to hypothetical 'ether'. Here a quote from 'Introduction to special relativity' by Robert Resnick is presented. '.......the experiment does not depend solely on an "absolute" velocity of earth through ether, but also depends on the changing velocity of the earth with respect to "ether". Such a changing motion through the "ether" would be easily detected and measured by the precision experiments, if there were an ether frame. The null result seems to rule out an ether (absolute) frame' [1].

Special relativity does not allow transportation of clocks for its inner consistency because motion of clocks and so change of motion might affect its reading. Even in practical life this is true. A good quality clock may stop by large acceleration. One may argue that this is a 'mechanical fault'. But to Einstein time is the reading of a set of synchronised clocks and therefore we a priori can not take the rating of clocks being independent of motion. According to Einstein's view a particular moving $S^{\prime}$ clock is observed against a background of mutually synchronised stationary $S$ frame clocks. In this situation identity of time standard in both frames has a specific meaning. If proper time interval between two events occurring at a particular $S^{\prime}$ clock is $\delta t^{\prime}=1$ then improper time interval between same two events but measured by two different $S$ frame clocks is not necessarily given by $\Delta t=1$ but by $\Delta t=k$. But if we reverse the perspective identically i.e, if we observe proper time interval $\delta t=1$ occurring at a particular $S$ clock then improper time interval between same two events but measured by two different synchronised $S^{\prime}$ clocks will be $\Delta t^{\prime}=k$. Identity of time standard or identity of clocks in both frames does not mean equality of time irrespective of the perspective as was taken granted by Newton but means equality of time interval under identical condition. This is precisely the view of Einstein's time. Once we accept Einstein's view, we are to give up the concept of absolute simultaneity of events observed from two different frames. To compare readings of clocks from two frames we have to ensure first that we are using identical clocks i.e. identical time standard in both frames under identical condition and then we have to find a universal way of synchronisation of clocks of both frames. Here a quote from the 'Meaning of Relativity' by Albert Einstein is presented. 'The theory of relativity is often criticized for giving, without justification, a central theoretical role to the propagation of light, in that it finds the concept of time upon the law of propagation of light. The situation, however, is somewhat as follows. In order to give physical significance to the concept of time, processes of some kind are required which enables relations to be established between different places. It is immaterial what kind of processes one chooses for such definition of time. It is advantageous, however, for the theory, to choose only those processes concerning which we know something certain. This holds for the propagation light in vacuum in a higher degree than for any other process which could be considered, thanks to the investigations of Maxwell and H.A.Lorentz'[2]. What did Einstein mean? The lecture given by Einstein is a very precise presentation and at the same time is too brief. It was only Einstein's intuition that he recognised the speed of light as the ultimate speed in nature. At the same time Michelson-Morley experiment failed to detect 'ether frame' where speed of light could take the value $\mathrm{C}=2.99792458 \times 10^{8} \mathrm{~ms}^{-1}$. So Einstein recognised that speed of light in free space as ultimate speed limit in any inertial frame. He could assert this as the first postulate of special relativity. As the second postulate he could write that we can use same numerical value of $c$ with same system of units in all inertial frames provided with identical length and time standards under identical circumstances. Invariance of c precisely contains these two things.

\section{MEANING OF INVARIANCE OF LAWS OF PHYSICS}

Einstein saw that Maxwell's laws of electrodynamics remain invariant under Lorentz transformation relations with same value of $c$ provided $\mathbf{E}$ and $\mathbf{B}$ field transform in a specific from one frame to another. Lorentz force relation $\mathbf{F} / \mathbf{q}=\mathbf{E}+\mathbf{v} \times \mathbf{B}$ is an experimental fact and Lorentz transformations can be obtained using only six postulates out of eight mentioned in the introduction. Invariance of the laws of electrodynamics (Maxwell's laws and Lorentz force law) and invariance of charge are not required for the derivation. It will be shown in following section that even the mass transformation relation can be obtained rigorously for a free particle using Lorentz transformation. Now using $E^{\prime}-E$ transformation $B^{\prime}-B$ transformation and charge invariance it is possible to obtain form invariant relation $\mathbf{F}^{\prime} / \mathbf{q}=\mathbf{E}^{\prime}+\mathbf{v}^{\prime} \times \mathbf{B}^{\prime}$ in frame $S^{\prime}$ if 'force' transforms in a definite way under Lorentz transformation. But the question is how Einstein understood that Maxwell's laws along with the Lorentz force law were correct laws of electrodynamics. The answer is that it was simply his genius that prompted him to guess 
that laws of electrodynamics are 'flawless' and must be form invariant in all inertial frames. So invariance of laws of electrodynamics came as postulates and gave a book keeping for force transformation. From the knowledge force and mass transformation Einstein reformulated Newton's second law in the form $\mathbf{F}=\mathrm{d}[\mathrm{m}(\mathrm{v}) \mathbf{v}] / \mathrm{dt}$. This is the covariant form. If the law were otherwise such as $\mathbf{F}=m(v) d v / d t$ then also under the condition $F=0$, linear momentum $\mathbf{p}=m(v) \mathbf{v}$ would remain constant for a single particle. However if we consider collision between two point particles and assume that there is no external force except the force of collision then net linear momentum $\mathbf{p}_{1}+\mathbf{p}_{\mathbf{2}}$ of the system remains constant. So we can write $d p_{1} / d t+d p_{2} / d t=0$. Obviously the first term may be guessed as $F_{1}$ i.e. force of collision on the first particle and $\mathbf{F}_{2}$ as force on second particle. This is a good guess about the form of force because net force $\mathbf{F}_{\mathbf{1}}+\mathbf{F}_{\mathbf{2}}$ must be zero in this case. But it is guess after all because $d p_{1} / d t+d p_{2} / d t=0$ and $F_{1}+F_{2}=\mathbf{0}$ do not necessarily prove that $\mathbf{F}=d p / d t$. But the knowledge in the transformation of force gives the unique choice $\mathbf{F}=\mathrm{dp} / \mathrm{dt}$ in place of $\mathbf{F}=\mathrm{m}(\mathrm{v}) \mathrm{d} \mathbf{v} / \mathrm{dt}$. So invariance of laws of physics although is a general axiom but for this, invariance of Maxwell's laws and Lorentz force law were taken as postulates to rectify mechanical law into covariant form. Therefore Einstein at once raised laws of electrodynamics to the status of invariant laws in all inertial frames. In fact invariance of Maxwell's laws only means that the forms of the laws must remain invariant from one frame to another provided the corresponding constants and variables are changed from unprimed to primed ones. It means if $c$ replaces $c^{\prime}, E$ replaces $E^{\prime}, B$ replaces $B^{\prime}, q$ replaces $q^{\prime}$ etc then same form in variant law must hold in all inertial frame under specific coordinate transformations. But he was Einstein who understood that it is the exact equality in the value of $c$ and $c^{\prime}$ through which the variables of the two frames can be compared at all. Without this equality we can never construct equivalent length and time standard under Lorentz transformation of coordinates. Similarly exact equality in the value of charge $q$ and $q^{\prime}$ as we saw is essential to obtain force transformation between the frames. So invariance of electrodynamical laws means not only form invariance but also exact equality of $c$ and $q$ value. So it is obvious that differential wave equation in free space takes invariant form with same $c$ in all inertial frames and it means that three dimensional spherical wave front propagation equations can be simultaneously used in all inertial frames. Einstein then rederived Lorentz transformation relations by defining space-time as a result of consistent way to synchronisation of clocks. Therefore, we can say that invariance of ' $c$ ' in all inertial frame is not a 'law proper' but is a definition for construction of identical time standards in all frames if and only if we can provide with the guarantee for identical length standards in all inertial frames. This is achieved by another postulate. This sixth postulate is that if two events occurring at two places are simultaneous in one inertial frame $S$, then these two events will also be simultaneous in a uniformly moving $S^{\prime}$ frame provided they occur on the perpendicular axis to the direction of relative motion of the frames. If it were otherwise the symmetry of the situation were lost. If this is taken as valid postulate then the seventh one necessarily follows. The length standard perpendicular to the direction of motion remains invariant in all inertial frames. Using this postulate together with invariance of $c$ the famous time dilation relation $\delta t^{\prime}=\left(1-\beta^{2}\right)^{1 / 2} \Delta t$ can be derived where $\beta=u / c$. For such simple derivation one can be referred to page $67[1]$.

\section{LORENTZ TRANSFORMATIONS FROM EQUIVALANCE OF LENGTH AND TIME STANDARD}

To derive full sets of transformation relations Einstein needed a eighth postulate in his theory. Space-time is homogeneous and isotropic in all inertial frames. So the transformation relations must involve only linear relation among space and time coordinates. So by Noether's theorem conservation principles for linear momentum, energy, angular momentum and energy are incorporated as postulates in the theory. Moreover, once we recognise that speed of light in free space is the ultimate speed in all frames, we are to give up the absolute meaning in the concept of simultaneity of events when the events are not located on the axis perpendicular to the direction of frame's velocity. If we consider relative motion of two inertial frames $S$ and $S^{\prime}$ along their coincident $x-x^{\prime}$ axis with the initial condition that at the coincident origins $\left(\mathrm{O}\right.$ and $\left.\mathrm{O}^{\prime}\right)$ configuration, both $\mathrm{O}$ and $\mathrm{O}^{\prime}$ clocks are synchronised to 'zero' setting and then time $\mathrm{t}^{\prime}$ of any event in $\mathrm{S}$ occurring at $\left(x^{\prime}, y^{\prime}, z^{\prime}\right)$ will have to be related with the time $t$ for the same event occurring at $(x, y, z)$ coordinate is $S$ by $t^{\prime}=a x+b t$. It is clear now that reverse transformation must be given by $t=-a x^{\prime}+b t^{\prime}$. The reason is that if we observe a particular moving $S^{\prime}$ clock against a set of synchronised $S$ clocks then proper time $\delta t^{\prime}=(a u+b) \Delta t=a u+b$ for improper interval $\Delta \mathrm{t}=1 \mathrm{~s}$. Similarly if a particular $\mathrm{S}$ frame clock is observed against a set of synchronised $S^{\prime}$ clocks then proper time $\delta \mathrm{t}=\{-\mathrm{a}(-\mathrm{u})+\mathrm{b}\} \Delta \mathrm{t}^{\prime}=\mathrm{au}+\mathrm{b}$ for improper $\mathrm{S}^{\prime}$ interval $\Delta \mathrm{t}^{\prime}=1 \mathrm{~s}$. This is the required condition for equivalence between clocks of two frames. Obviously $a u+b=\left(1-\beta^{2}\right)^{1 / 2}$. Once this is done there happen two things. Simultaneity of events along the axis of frame's motion becomes relative and therefore space and time looses their independent existence but emerges as single continuum where energy-momentum together appears in the corresponding transformation realiotns. At the second place whole set of $x^{\prime}-x$ and $t^{\prime}-t$ transformation relation are readily obtained. Using $t^{\prime}=a x+b t$ and $t=-a x^{\prime}+b t^{\prime}$ we find

$$
\begin{aligned}
& x^{\prime}=b\left\{x-\left(1-b^{2}\right) t / a b\right\} \\
& x=b\left\{x^{\prime}+\left(1-b^{2}\right) t^{\prime} / a b\right\}
\end{aligned}
$$

If we observe motion of a particular $S^{\prime}$ clock located at particular primed coordinate then its equation of motion in $S$ frame is given by

$$
x^{\prime} / b+\left(1-b^{2}\right) t / a b=x
$$

i.e.

$$
\mathrm{X}=\mathrm{x}_{0}+\mathrm{ut}
$$

where,

$$
\left(1-b^{2}\right) / a b=u
$$

We already had $a u+b=\left(1-\beta^{2}\right)^{1 / 2}$ for equivalence of $S$ and $S^{\prime}$ clocks where length standard in a direction perpendicular to the direction of frame's motion remains invariant. Solving the above two relations for $a$ and $b$ we get $b=1 /\left(1-\beta^{2}\right)^{1 / 2}$ and 
$a=-\beta / c\left(1-\beta^{2}\right)^{1 / 2}$ with these values we see that $x^{\prime}-x$ or $t^{\prime}-t$ relations and the reverse transformations are exactly Lorentz transformation given by

$$
\begin{aligned}
& x^{\prime}=b(x-u t) \\
& t^{\prime}=b\left(t-u x / c^{2}\right) \\
& x=b\left(x^{\prime}+u t^{\prime}\right) \\
& t=b\left(t^{\prime}+u x^{\prime} / c^{2}\right)
\end{aligned}
$$

Einstein [4] obtained the transformation relations not by the simple argument as given above but by using clock synchronisation rule $t_{B}-t_{A}=t_{A}^{\prime}-t_{B}$ and by length measuring rule $2(A B)=c\left(t_{A}^{\prime}-t_{A}\right)$ using same $c$ in any inertial frame. Here $t_{A}$ is the pulse emission time and $t_{A}{ }^{\prime}$ is the same pulse receiving time at $A$ after reflection from $B$ clock. With this rule he assumed that axis perpendicular to the relative motion between the frames remains perpendicular in all frames and spacetime is homogeneous.

Finally it becomes now necessary to check equivalence of length standard in direction of motions of frames. It means if a moving rod's two ends are measured simultaneously from frame $S$ to produce a value $\Delta l=1$ then in the frame $S^{\prime}$ where the rod is at rest, the 'proper' length will produce $\delta l^{\prime}=l^{\prime}$ with two ends measured at different $S^{\prime}$ time. Obviously in such case $\Delta \mathrm{t}=0$ but $\Delta \mathrm{t}^{\prime}=\mathrm{t}_{2}^{\prime}-\mathrm{t}_{1}^{\prime}=\mathrm{a}\left(\mathrm{x}_{2}-\mathrm{x}_{1}\right)=\mathrm{a} \Delta \mathrm{l}=\mathrm{a}$ and $\mathrm{x}_{2}^{\prime}-\mathrm{x}_{1}^{\prime}=\mathrm{l}^{\prime}=\mathrm{b}\left(\mathrm{x}_{2}-\mathrm{x}_{1}\right)=\mathrm{b} .1=\mathrm{b}$. If the situation is reversed i.e., $\Delta \mathrm{l}^{\prime}=1$ for a moving rod in $S^{\prime}$ where two ends are measured simultaneously $(\Delta t=0)$ then $\delta l$ i.e., proper length in $S$ frame must equals $I^{\prime}$. Now $\Delta \mathrm{t}=\mathrm{t}_{2}-\mathrm{t}_{1}=-\mathrm{a}\left(\mathrm{x}_{2}^{\prime}-\mathrm{x}_{1}^{\prime}\right)=-\mathrm{a} \Delta \mathrm{l}^{\prime}$. As stationary clocks in both frames are identical so $\Delta \mathrm{t}^{\prime}=-\mathrm{a} \Delta \mathrm{l}^{\prime}$ must equals $-\mathrm{a}$ under identical experiments as mentioned if $\Delta l^{\prime}=1$. Similarly from $x-x^{\prime}$ relation we get, $x_{2}-x_{1}=l^{\prime}=b\left(x_{2}^{\prime}-x_{1}^{\prime}\right)=b \Delta l^{\prime}=b$, if equivalence of length standard in the direction of frame's relative velocity is to be guaranteed. It simply means $\Delta l^{\prime}=1$ as before. It means that not only clocks of both frames are equivalent in terms of proper time but also they are equivalent in terms of improper time interval.

\section{TRANSFORMATION RELATION FOR MASS AND LINK BETWEEN CONSERVATIION AND INVARIANCE OF CHARGE}

To obtain mass transformation relation independently we may proceed in the following way.

Let $m_{0}$ is rest mass of a free particle in proper frame $S^{\prime}$ and the corresponding moving mass in frame $S$ is $m$. In $S$ frame each component of momentum is constant.

$$
\mathrm{p}_{\mathrm{\alpha}}=\mathrm{mdx}_{\mathrm{\alpha}} / \mathrm{dt}=\text { constant }
$$

Here, $x_{1}=x, x_{2}=y, x_{3}=z, x_{4}=t$. For $\alpha=1,2,3$ we get momentum conservation along respective axis and for $\alpha=4$ we get $\mathrm{m}=$ constant. If $\mathrm{dt}$ is proper time interval then it is invariant. So we can write

$$
\begin{aligned}
p_{\alpha} & =m\left(d x_{\alpha} / d t^{\prime}\right)\left(1-\beta^{2}\right)^{1 / 2}=\text { constant } \\
& =\sum C_{\alpha \beta}^{\prime \prime} m\left(d x^{\prime \prime}{ }_{\beta} / d t^{\prime}\right)\left(1-\beta^{2}\right)^{1 / 2}=\text { constant } \\
p_{\alpha} & =\sum C^{\prime \prime}{ }_{\alpha \beta} A_{\beta}^{\prime \prime}{ }_{\beta}=\text { constant }
\end{aligned}
$$

Here $C^{\prime \prime}{ }_{\alpha \beta}$ are transformation coefficients of coordinates from $S$ frame to another frame $S^{\prime \prime}$ and are constants by virtue of Lorentz transformations.

As $C^{\prime \prime}{ }_{\alpha \beta}$ are all constants so $A_{\beta}^{\prime \prime}$ is constant. So we have

$$
A^{\prime \prime}=m\left(d x^{\prime \prime}{ }_{\beta} / d t^{\prime}\right)\left(1-\beta^{2}\right)^{1 / 2}=\text { constant }
$$

In $S^{\prime \prime}$ we but have

$$
\begin{aligned}
\mathrm{p}^{\prime \prime} & =\mathrm{m}^{\prime \prime}\left(\mathrm{dx}_{\beta}{ }_{\beta} / \mathrm{dt}^{\prime \prime}\right) \\
& =\mathrm{m}^{\prime \prime}\left(\mathrm{dx^{ \prime \prime }}{ }_{\beta} / \mathrm{dt}\right)\left(1-\beta^{/ / 2}\right)^{1 / 2}=\text { constant }
\end{aligned}
$$

Here $\beta=u / c$ and $\beta^{\prime \prime}=u^{\prime \prime} / c$.

Hence we can write for any arbitrary $\lambda$

$$
\begin{array}{ll} 
& \mathrm{A}_{\beta}^{\prime \prime}-\lambda \mathrm{p}^{\prime \prime}{ }_{\beta}=0 \\
\text { or, } & {\left[\mathrm{m}\left(1-\mathrm{u}^{2} / \mathrm{c}^{2}\right)^{1 / 2}-\lambda \mathrm{m}^{\prime \prime}\left(1-\mathrm{u}^{\prime / 2} / \mathrm{c}^{2}\right)^{1 / 2}\right] \mathrm{dx}{ }^{\prime \prime}=0} \\
\text { or, } & \mathrm{m}\left(1-\mathrm{u}^{2} / \mathrm{c}^{2}\right)^{1 / 2}-\lambda \mathrm{m}^{\prime \prime}\left(1-\mathrm{u}^{1 / 2} / \mathrm{c}^{2}\right)^{1 / 2}=\text { invariant }
\end{array}
$$

If we put $u=0$ we get $m_{0}$ as the invariant. Hence we get $m=m_{0} /\left(1-\beta^{2}\right)^{1 / 2}$ and $m^{\prime \prime}=m_{0} / \lambda\left(1-\beta^{/ / 2}\right)^{1 / 2}$. If expression for moving mass is covariant then $\lambda=1$.

Now let us turn attention to charge invariance. It is a precious invariant quantity. Apart from the necessity to obtain the force transformation relation, there remains another compulsion for charge invariance. It directly follows from charge conservation principle. It is established fact that conserved quantities may not be invariants. Net linear momentum, net relativistic mass or energy is such examples. But charge conservation is a different story. If this principle were not a good one, mere motion of charge could create or destroy charge or could alter charge value like mass. No such violation is 
found yet. If charge remains invariant of motional state then it is clear that 'stationary charge' or 'proper charge' and 'moving charge' must have same value in a frame. Dimension of charge evaluated from Coulomb's law in CGS esu is $\left[\mathrm{M}^{1 / 2} \mathrm{~L}^{3 / 2} \mathrm{~T}^{-1}\right]$. If two inertial frames are compared with identical length, time and mass standard under identical circumstances then same charge value in all frames can of course be used using same system of units.

Charge invariance is a direct outcome of charge conservation. But can we expect any violation in this principle? Feynman answers 'The laws of physics have no answer to this question: "what happens if a charge is suddenly created at this pointwhat electromagnetic effects are produced?" No answer can be given because our equations say it doesn't happen. If it were to happen, we would need laws, but cannot say what they would be. We have not had the chance to observe without charge conservation behaves' [3]. After all we had to abandon two very noble conservation laws namely mass conservation and energy conservation separately following relativistic mechanics to fit observed data from nuclear reactions. So, more and more precise experiments might cast doubt on charge conservation principle. But chance is too thin in present day context.

\section{DOPPLER EFFECT FROM OF MOVING CLOCK}

Let us consider an $S^{\prime}$ clock emitting a continuous monochromatic wave train of angular frequency $\omega^{\prime}$ with propagation direction cosines $\mathrm{I}^{\prime}, \mathrm{m}^{\prime}, \mathrm{n}^{\prime}$ with respect to $S^{\prime}$ clock. The same clock is observed as a moving clock from $S$ frame with corresponding values $\omega, \mathrm{I}, \mathrm{m}, \mathrm{n}$. Here $\mathrm{I}, \mathrm{m}, \mathrm{n}$ are direction cosines of wave propagation from instantaneous position of $\mathrm{S}$ clock. If the clock reads a proper time interval $\delta t^{\prime}$ in $S^{\prime}$ then the phase angle proper lapsed in the $S^{\prime}$ clock is given by $\omega^{\prime} \delta t^{\prime}$. The question is what the phase angle lapsed in the moving clock observed from $S$ frame is. The answer is not certainly $\omega \Delta t$ where $\left(1-\beta^{2}\right)^{1 / 2} \Delta t=\delta t$. The moving clock has traversed the rectilinear distance $u \Delta t$ in $S$ frame. Therefore, when we have to find phase angle, we have to consider not only the term $\omega \Delta t$ but also the initial and final location of moving clock. If a continuous wave train were emitted from fixed initial position, phase angle of wave lapsed would certainly be $\omega \Delta t$ at the initial position. But after time $\Delta t$ the clock has advanced $\mathrm{u} \Delta \mathrm{t}$ distance and still is emitting monochromatic with direction cosines I, $\mathrm{m}, \mathrm{n}$. So the phase of wave emitted at new location is given by $\omega \Delta t-\mathbf{k} . \Delta \mathbf{x}$. Here $\mathbf{k}$ is a wave vector and is given by $(\mathbf{i} \mid+\mathbf{j m}+\mathbf{k n}) \mathrm{k}$ and $\Delta \mathbf{x}=\mathbf{i u} \Delta \mathrm{t}$. So phase angle of moving clock or of the wave emitted from the moving clock in the new location is $\omega \Delta t-l k u \Delta t=\omega \Delta t(1-\mid \beta)$ where $\omega / k=c$. Obviously the phase angle travelled in the moving clock equals the difference of final phase in new location and initial phase say zero phase at initial position. As the clocks are identical and the phase angle is a dimensional number so $\omega^{\prime} \delta t^{\prime}=\omega \Delta t(1-\mid \beta)$. Hence $\omega^{\prime}=\omega(1-\mid \beta) /\left(1-\beta^{2}\right)^{1 / 2} i$. e. $v^{\prime}=v(1-\mid \beta) /\left(1-\beta^{2}\right)^{1 / 2}$. This is exactly the Doppler formula where $v^{\prime}$ is the frequency of light emitted from a stationary source in $S^{\prime}$ frame and $v$ is the frequency of light emitted by a moving source as observed from $S$ frame and is detected by stationary detector in $S$ frame. This result is remarkable in the sense that we not only obtained Doppler formula but also found what does phase of a clock mean.

\section{CONCLUDING REMARKS}

Special Relativity was constructed in such a way that all the three base quantities can be used with identical units (kg, $\mathrm{m}$ and s) in all inertial frames under identical conditions so that one can compare values from one frame to the others. This was a stupendous task and required Einstein's genius. These identical standards are not given automatically by nature as was supposed by Newton but is a creation of a genius mind. Atoms and nuclei are endowed with their characteristics frequency, wavelength of radiation they emit and have characteristics mass in their proper or rest frame. Therefore we can relate these values as observed from any other frame with those in proper frames if and only if we have identical standards of base quantities. Einstein consciously found the way but at the cost of sacrificing universal time. Einstein wrote -'Turning to the theory of relativity itself, I am anxious to draw attention to the fact that this theory is not speculative in origin; it owes its invention entirely to the desire to make physical theory fit observed fact as well as possible. We have here no revolutionary act but the natural continuation of a line that can be traced through centuries. The abandonment of certain notions connected with space, time and motion hitherto treated as fundamental must not be regarded as arbitrary, but only as conditioned by observed facts' [5]. We of course understand today what a revolutionary work it was that changed physics forever. This is a frame work of eight postulates upon which Einstein built his theory. But the true view of the meaning of the invariance of $c$ is generally missing in scientific literatures and in texts even after 110 years from the birth of the theory.

\section{.ACKNOWLEDGMENTS}

Author acknowledges the contribution of Sri Debasis Dev, Assistant Research Officer of Hydraulics division of River Research Institute, West Bengal, India for preparing and formatting the manuscript and his valuable suggestion.

\section{REFERENCES}

[1] Introduction to Special Relativity, First U.S. Edition 1968, Sixth Wiley Eastern Reprint 1985, page 26, sec-1.5, ISBN 0852267614

[2] The Meaning of Relativity, Albert Einstein, Princeton University Press 1922, Lecture-II: The theory of special relativity, page $29-30$

[3] The Feynman lectures on physics vol.2, chapter 17: the laws of Induction p-253(c) 1964 Addison- Wesley Publishing Company, Inc, $15^{\text {th }}$ Narosa Publishing House reprint 2008. ISBN81-85015-83-X

[4] On The Electrodynamics Of Moving Bodies , Albert Einstein, Annalen der Physik 17(1905): 891921 ,einsteinpapers.press.princeton.edu/vol2-trans/154 
[5] On the theory of Relativity; Albert Einstein. Lecture at King's College, London, 1921. Published in 'Mein Weltbild, Amsterdam: Querido Verlag, 1934

\section{Author' biography with Photo}

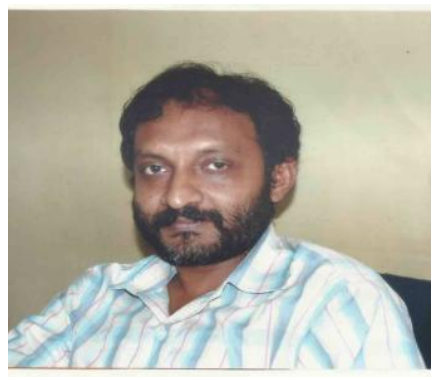

Graduated with Physics and now currently is involved in hydraulic research work as Assistant Research Officer at River Research Institute, West Bengal, Pin-741246, India. Published (1) Special Relativity Contracdicted by Oscillation-IJSER (ISSN 2229 5518), VOL-5, ISSUE 2, 2014, (2) Planck's Quantum Hypothesis and Concepts of Mass form Special Relativity- IJSER (ISSN 2229 5518), VOL-6, ISSUE 6, 2015, (3) Logarithmic Velocity Profile for Turbulent Flow in Straight Rough Pipe and Evaluation of Karmann Constant with Bouindary Layer Reynolds Number- A Copmplete Solution -IJSER (ISSN 2229 5518), VOL-7, ISSUE 2, 2016.

Ph. No. +919836767097 